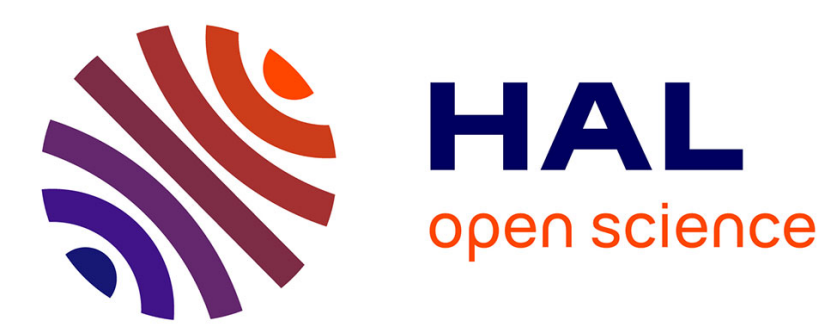

\title{
Impact des politiques climatiques sur le prix du carbone et les marchés de l'énergie
}

Odile Blanchard, Patrick Criqui, Alban Kitous, Silvana Mima

\section{To cite this version:}

Odile Blanchard, Patrick Criqui, Alban Kitous, Silvana Mima. Impact des politiques climatiques sur le prix du carbone et les marchés de l'énergie. Revue d'économie financière, 2006, 83, p. 91-113. halshs-00006165

\section{HAL Id: halshs-00006165 https://shs.hal.science/halshs-00006165}

Submitted on 25 Nov 2005

HAL is a multi-disciplinary open access archive for the deposit and dissemination of scientific research documents, whether they are published or not. The documents may come from teaching and research institutions in France or abroad, or from public or private research centers.
L'archive ouverte pluridisciplinaire HAL, est destinée au dépôt et à la diffusion de documents scientifiques de niveau recherche, publiés ou non, émanant des établissements d'enseignement et de recherche français ou étrangers, des laboratoires publics ou privés. 


\title{
Impact des politiques climatiques sur le prix du carbone et les marchés de l'énergie
}

\author{
Odile Blanchard*, Patrick Criqui*, Alban Kitous ${ }^{\circ}$, Silvana Mima* \\ *Laboratoire d'Economie de la Production et de l'Intégration Internationale- \\ Département Energie et Politiques de l'Environnement, Grenoble (LEPII-EPE) \\ $\circ$ Enerdata, Grenoble
}

novembre 2005

\section{Auteurs :}

Odile Blanchard,

Maître de Conférences

Laboratoire d'Economie de la Production et de l'Intégration Internationale-

Département Energie et Politiques de l'Environnement, Grenoble (LEPII-EPE), BP 47, 38040

Grenoble Cedex 9

Tel 0456528584

Fax 0456528571

Email : Odile.Blanchard@upmf-grenoble.fr

Patrick Criqui,

Directeur de Recherche au CNRS

Laboratoire d'Economie de la Production et de l'Intégration Internationale-

Département Energie et Politiques de l'Environnement, Grenoble (LEPII-EPE), BP 47, 38040

Grenoble Cedex 9

Tel 0456528573

Fax 0456528571

Email : Patrick.Criqui@upmf-grenoble.fr

Alban Kitous

Ingénieur Economiste

ENERDATA

2 Avenue de Vignate

38610 GIERES

FRANCE

Tél 0476422546

Fax 0476516145

alban.kitous@enerdata.fr

Silvana Mima

Chercheuse

Laboratoire d'Economie de la Production et de l'Intégration Internationale-

Département Energie et Politiques de l'Environnement, Grenoble (LEPII-EPE), BP 47, 38040

Grenoble Cedex 9

Tel 0456528589

Fax 0456528571

Email : Silvana.Mima@upmf-grenoble.fr 


\section{Résumé :}

Ce papier vise à analyser les interactions entre les politiques de stabilisation des concentrations de gaz à effet de serre dans l'atmosphère et les fondamentaux de la scène pétrolière mondiale, à partir de simulations du modèle POLES. La « valeur du carbone » est l'artifice de modélisation qui synthétise l'intensité des politiques climatiques. Elle constitue le signal qui déclenche les investissements de réduction des émissions de gaz à effet de serre, investissements socialement responsables au regard du défi climatique. La comparaison d'un scénario énergétique tendanciel avec un scénario de division par quatre des émissions des pays industrialisés à l'horizon 2050 permet de montrer que la conduite de politiques climatiques très ambitieuses permet à la fois de limiter le changement climatique et de gérer la question de l'épuisement des ressources mondiales d'hydrocarbures.

\section{Abstract :}

\section{The impact of climate policies on the carbon price and the energy markets}

Based on simulations of the POLES model, this paper aims to analyse the interactions between policies to stabilize greenhouse gas concentrations in the atmosphere and the fundamentals of the world oil market. The « carbon value » is the modeling device that reflects the relative harshness of climate policies. It represents the signal that triggers socially responsible investments addressing the climate challenge, ie greenhouse gas abatement investments. We compare a Reference energy scenario and a scenario of emission reductions by a factor four in industrialized countries by 2050. The results show that very ambitious climate policies allow both to mitigate climate change and to manage the depletion of world oil resources.

\section{Codes JEL : Q58, Q41}




\title{
Impact des politiques climatiques sur le prix du carbone et les marchés de l'énergie
}

\author{
Odile Blanchard*, Patrick Criqui*, Alban Kitous ${ }^{\circ}$, Silvana Mima* \\ *Laboratoire d'Economie de la Production et de l'Intégration Internationale- \\ Département Energie et Politiques de l'Environnement, Grenoble (LEPII-EPE) \\ $\circ$ Enerdata, Grenoble
}

Le réchauffement climatique global et ses impacts écologiques et socio-économiques constituent un des défis majeurs pour l'humanité au $21^{\text {ème }}$ siècle. L'augmentation de la température moyenne de la terre et les probables bouleversements climatiques associés seront d'autant plus forts que les émissions de gaz à effet de serre (GES) liées aux activités humaines croîtront de manière importante. En effet, les GES étant des polluants-stocks, leur concentration dans l'atmosphère, à l'origine du réchauffement, continueront d'augmenter tant que les émissions ne seront pas ramenées au niveau de la capacité d'absorption naturelle du « système terre », c’est à dire à environ un tiers du niveau actuel des émissions.

Depuis le début des années quatre vingt-dix, la communauté internationale a pris conscience de la nécessité de lutter contre le changement climatique. La quasi-totalité des pays de la planète a ratifié la Convention Cadre sur les Changements Climatiques, dont l'objectif ultime est de « stabiliser les concentrations de gaz à effet de serre dans l'atmosphère à un niveau qui empêche toute perturbation anthropique dangereuse du système climatique » (article 2, UNFCCC, 1992). De très nombreux pays ${ }^{1}$ ont ensuite ratifié le Protocole de Kyoto qui impose aux pays industrialisés de réduire globalement de 5 \% leurs émissions de GES en 2008-2012 par rapport à 1990 (UNFCCC, 1997).

Cependant chacun s’accorde à souligner que les engagements pris dans le Protocole de Kyoto ne sont que la toute première étape de la bifurcation à opérer dans les prochaines décennies vers des trajectoires permettant de stabiliser les concentrations. Dans plusieurs pays européens on évoque aujourd'hui l'objectif d'une division par trois ou quatre des émissions dans les pays industrialisés, qui devrait être accompagnée d'un fort ralentissement de la hausse des émissions dans les pays en développement ${ }^{2}$. Les profils d'émission mondiaux résultants permettraient alors de stabiliser la concentration des émissions de GES à moins de deux fois le niveau antérieur à l'ère industrielle, entre 450 et 550 ppmv $^{3}$, contre 270 en 1800 (Criqui et al., 2003).

Par ailleurs, dans les prochaines décennies le prix du pétrole devrait s’inscrire sur une trajectoire très supérieure en niveau à celle des vingt dernières années. Ceci du fait des tensions sur les marchés qui vont résulter de la combinaison d'une forte pression de la demande mondiale et d'une offre conventionnelle globale appelée à décliner inexorablement, par insuffisance des nouvelles ressources après le passage d'un plafond de production. C'est le phénomène du « peak oil » identifié aujourd'hui non seulement par des géologues et des ONG environnementales, mais aussi par certaines des grandes compagnies internationales (Porter ,1995 ; Wells, 2005).

\footnotetext{
${ }^{1} 156$ « Parties » ont ratifié le Protocole de Kyoto (mise à jour septembre 2005)

${ }^{2}$ A son allocution d'ouverture du débat national sur les énergies, en mars 2003, le premier ministre français, M.Raffarin, s’est prononcé pour une division par quatre des émissions des pays industrialisés. A la même époque, le premier ministre britannique, M. Blair, a évoqué une réduction de 60\%, en s’appuyant sur le «White Paper » du Département du Commerce et de l’Industrie (DTI, 2003).

${ }^{3}$ particules par million en volume
} 
Ce papier vise à analyser, dans une perspective systémique, les interactions entre les politiques de stabilisation des concentrations de GES dans l'atmosphère et les fondamentaux de la scène pétrolière mondiale. Nous montrerons que la conduite de politiques climatiques ambitieuses permet à la fois de réduire les émissions de GES (et donc de sauvegarder le climat global), et de diminuer les tensions sur le marché pétrolier (et donc de limiter la hausse du prix du pétrole). La « valeur du carbone (ou valeur-carbone) » utilisée dans les dispositifs de modélisation est un artifice qui permet de synthétiser dans une variable unique le signal à fournir pour déclencher les investissements de réduction des émissions de GES. Elle permet de mesurer l'effort à entreprendre pour limiter les émissions et rend bien compte de l'effort d'investissement socialement responsable qui permettrait la sauvegarde du climat de la planète.

La première partie de ce papier est consacrée à une description rapide du modèle énergétique mondial POLES qui est utilisé ici pour simuler les politiques climatiques et leurs impacts sur les marchés énergétiques. La deuxième partie présente les hypothèses et principaux résultats du scénario énergétique « tendanciel » à l’horizon 2050. Enfin, la troisième partie montre l'incidence de l'introduction d'une taxe-carbone élevée sur les émissions de gaz carbonique (CO2) et sur le prix du pétrole, dans un scénario visant à diviser par quatre les émissions des pays industrialisés en 2050. $\mathrm{CO}_{2}$

\section{POLES, un modèle mondial d'équilibre partiel pour l'énergie}

Le modèle POLES (Prospective Outlook on Long term Energy Systems) est un modèle d'équilibre partiel du système énergétique mondial (Criqui et Kouvaritakis, 2000 ; Criqui et Viguier, 2000). Il fournit une prospective détaillée à l’horizon 2050, en pas annuels, pour les grands pays ou régions (46 au total), avec simulation de la demande d'énergie par secteur, de l’offre et des prix internationaux des énergies primaires, des technologies énergétiques nouvelles et renouvelables, ainsi que des grands systèmes de conversion (électrique et, pour le long terme, hydrogène). Le progrès technique, au sein du secteur de l'énergie, est explicitement pris en compte, soit de manière exogène, soit par l'endogénéisation des effets d'expérience et d'apprentissage pour les différentes technologies (figure 1). 


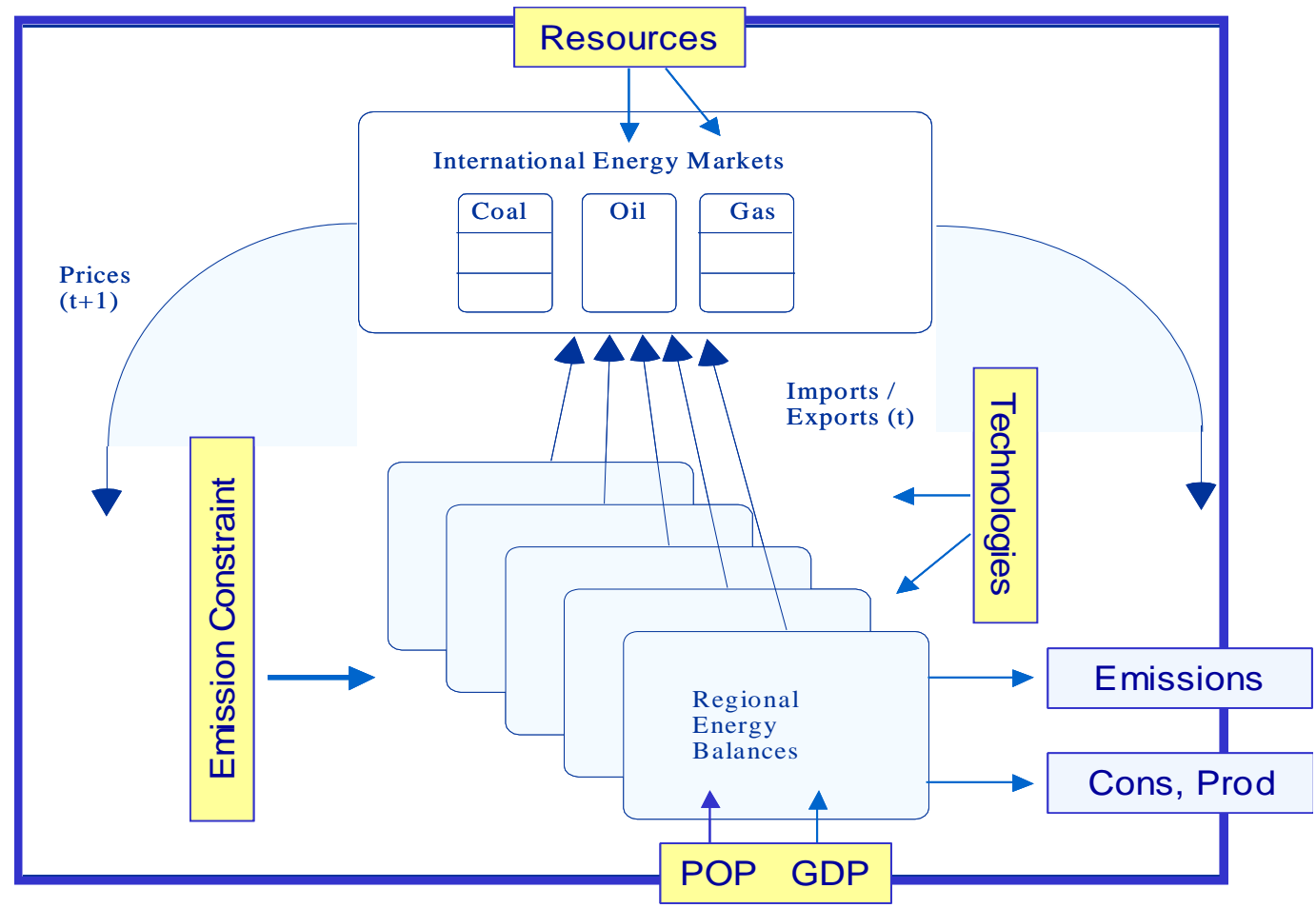

Ce modèle fournit ainsi un cadre cohérent pour l'analyse des dynamiques énergieenvironnement. En amont il prend en compte des scénarios exogènes de croissance économique et démographique par région, ainsi que les contraintes de ressource pour le pétrole et le gaz. En aval, il permet de calculer les émissions de GES dégagées par la combustion des combustibles fossiles et de calculer les coûts marginaux de réduction des émissions des différents pays ou régions. Il permet alors de simuler différents scénarios de contraintes d'émission, d'analyser les effets de l'introduction d'une taxe carbone ou d'étudier les systèmes d'échanges de quotas d'émission négociables.

\section{Scénario tendanciel à 2050 : vers un doublement des consommations mondiales d'énergie et des émissions de $\mathrm{CO} 2$}

La construction d'un scénario tendanciel fournit une image possible du monde de l'énergie en 2050, sur la base d'hypothèses exogènes plausibles compte-tenu des tendances et des contraintes actuellement identifiées. Il ne s’agit pas d'une prévision, mais simplement d'une projection énergétique cohérente pour un futur possible. C'est à partir de cette projection que peuvent ensuite être élaborés des scénarios de politiques actives, par exemple en matière de lutte contre le changement climatique. Le scénario « Stabilisation 450 - Facteur 4 » de la troisième partie en est une illustration. Nous présenterons tout d'abord les hypothèses exogènes sur lesquelles repose le scénario tendanciel, puis ses principaux résultats en termes de consommations d'énergie, de prix du pétrole et d'émissions de GES.

\subsection{Les hypothèses exogènes du scénario tendanciel}

Le tableau 1 résume les hypothèses macro-économiques et démographiques retenues pour l'élaboration du scénario tendanciel. Elles résultent d'hypothèses définies pour chacune des 
régions spécifiquement prises en compte dans le modèle. Le ralentissement de la croissance démographique mondiale provient par exemple de dynamiques contrastées : sur 2030-2050, la population de quatre régions décroît ${ }^{4}$, tandis que celle des autres régions continue d'augmenter, quoique plus lentement qu'auparavant. De même, la multiplication par quatre du PIB mondial d'ici 2050 reflète une croissance économique beaucoup plus rapide dans les pays en développement que dans les pays industrialisés, mais avec également une décélération très marquée de la croissance en Chine, due au vieillissement attendu de la population. Le scénario tendanciel n’est donc pas un scénario de pure extrapolation.

Tableau 1 : hypothèses démographiques et macro-économiques exogènes

\begin{tabular}{|c|c|c|c|c|c|c|c|c|}
\hline \multirow{2}{*}{\begin{tabular}{|l|} 
Indicateurs Clefs \\
\end{tabular}} & \multirow[b]{2}{*}{1990} & \multirow[b]{2}{*}{2001} & \multirow[b]{2}{*}{2010} & \multirow[b]{2}{*}{2030} & \multirow[b]{2}{*}{2050} & \multicolumn{3}{|c|}{ Croissance (\%/an) } \\
\hline & & & & & & $1990 / 10$ & $2010 / 30$ & $30 / 50$ \\
\hline Population (Milliards) & 5.2 & 6.1 & 6.8 & 8.1 & 8.9 & $1.3 \%$ & $0.9 \%$ & $0.5 \%$ \\
\hline PIB (M€) & 29880 & 42224 & 59524 & 105930 & 164090 & $3.5 \%$ & $2.9 \%$ & $2.2 \%$ \\
\hline PIB par habitant $(€)$ & 5697 & 6907 & 8764 & 13107 & 18513 & $2.2 \%$ & $2.0 \%$ & $1.7 \%$ \\
\hline
\end{tabular}

Le PIB est exprimé en $€$ constants de 1999

Source : POLES

Par ailleurs, compte tenu d'une prise de conscience croissante de la nécessité de lutter contre le changement climatique, il apparaît probable que, dans les décennies à venir, les politiques énergétiques intégreront des actions pour limiter les émissions de GES, au moins « a minima ». L'introduction d'une valeur-carbone sur les émissions de CO2 liées à l'utilisation de combustibles fossiles, différenciée par région, est l'artifice utilisé dans le modèle POLES pour refléter ces actions. Elle ne suppose pas un accord international sur le climat mais entend simplement représenter des politiques climatiques « a minima », différenciées selon les régions.

Dans ce cadre, hypothèse est faite que l'Europe demeure la région montrant la volonté d'action la plus forte. La valeur-carbone de cette région est fixée de façon exogène à $10 € / t C O 2$ en 2010 (pour l'ensemble de l'économie) puis elle augmente linéairement jusqu'à $30 € /$ tCO2 en 2050. Les autres pays industrialisés sont supposés adopter des mesures plus modérées. Ceci se traduit par une valeur-carbone inférieure de moitié à celle de l'Europe ( $5 € /$ tCO2 en 2010) augmentant linéairement pour atteindre $15 €$ en 2050. Enfin hypothèse est faite que les pays en développement n'engagent des actions qu'après 2010 et qu'ils rejoignent en 2050 l'effort des autres pays industrialisés. Cette hypothèse se traduit par une taxe-carbone qui est nulle jusqu'en 2010 puis augmente progressivement jusqu’à $15 € /$ tCO2 en 2050.

\subsection{Perspectives énergétiques à 2050}

Compte tenu des hypothèses exogènes adoptées pour le scénario tendanciel, la consommation d'énergie mondiale est plus que multipliée par deux en cinquante ans (figure 2). Alors que le PIB est lui-même multiplié par quatre dans le même temps, cela signifie déjà une diminution significative de l'intensité énergétique du PIB mondial, qui s'explique par la combinaison de changements structurels dans l'économie et une hausse significative des prix de l'énergie (voir infra). Toutes les sources d'énergie primaire progressent en niveau, qu'elles soient d'origine fossile (charbon, gaz, pétrole) ou non fossile (renouvelables, nucléaire). Les énergies renouvelables et le nucléaire occupent une place croissante dans le bilan énergétique, car leur expansion est rapide, en particulier après 2030.

\footnotetext{
${ }^{4}$ Il s’agit de l'Europe, de l'Australasie, de la Communauté des Etats Indépendants et de la Chine
} 
Mais les énergies fossiles représentent encore près de $70 \%$ du bilan mondial en 2050 (contre 80 \% aujourd'hui), ce qui laisse déjà entrevoir des niveaux d'émissions de CO2 élevés (voir infra). Dans une large mesure, le scénario tendanciel est un scénario de retour du charbon. Cette source, abondante au plan mondial maintient sa part de marché, tandis que le pétrole et le gaz perdent globalement des parts sur la période, sous l'effet des contraintes de ressource et des prix élevés qui en découlent (voir infra).

Figure 2. Consommation totale mondiale et répartition par sources (2001-2050), scénario tendanciel

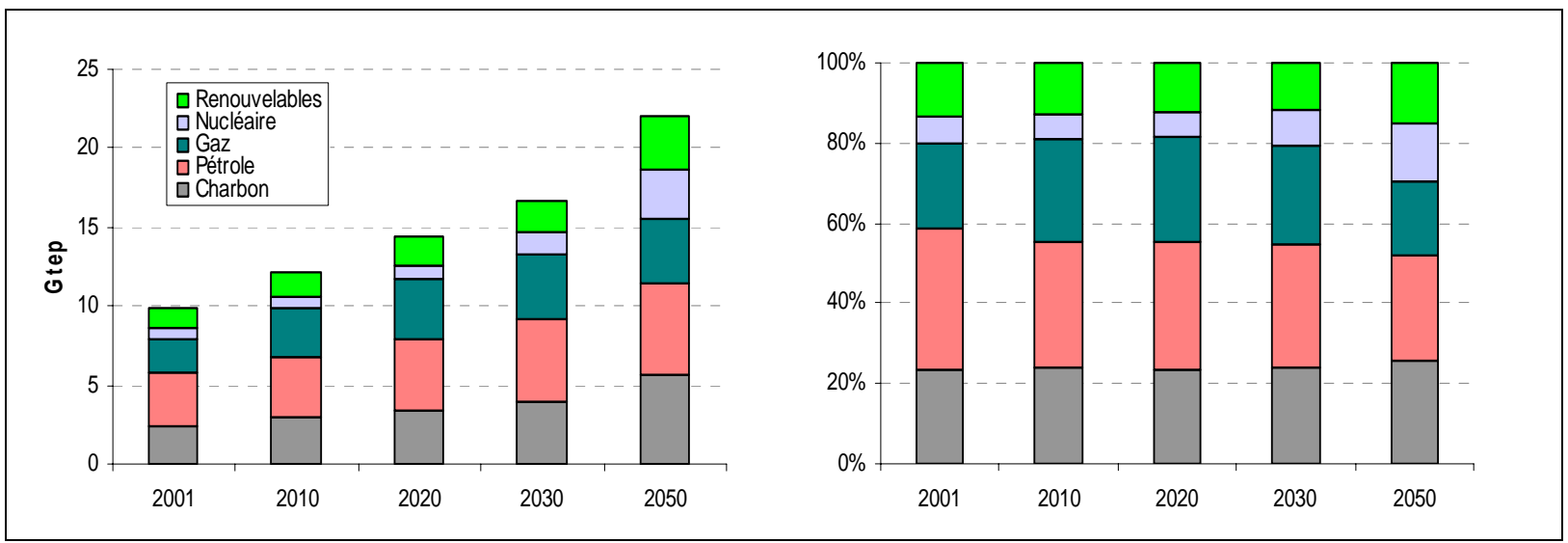

Gtep = milliards de tonnes-équivalent-pétrole

Source : modèle POLES

En termes de répartition régionale (figure 3), les pays industrialisés représentent environ $60 \%$ des consommations en 2001, et seulement un peu plus du tiers en 2050. L'augmentation des consommations d'énergie est beaucoup plus rapide dans les pays en développement, sous l'effet de dynamiques démographiques et économiques plus fortes.

Figure 3 : répartition géographique de la consommation mondiale (2001-2050), scénario tendanciel

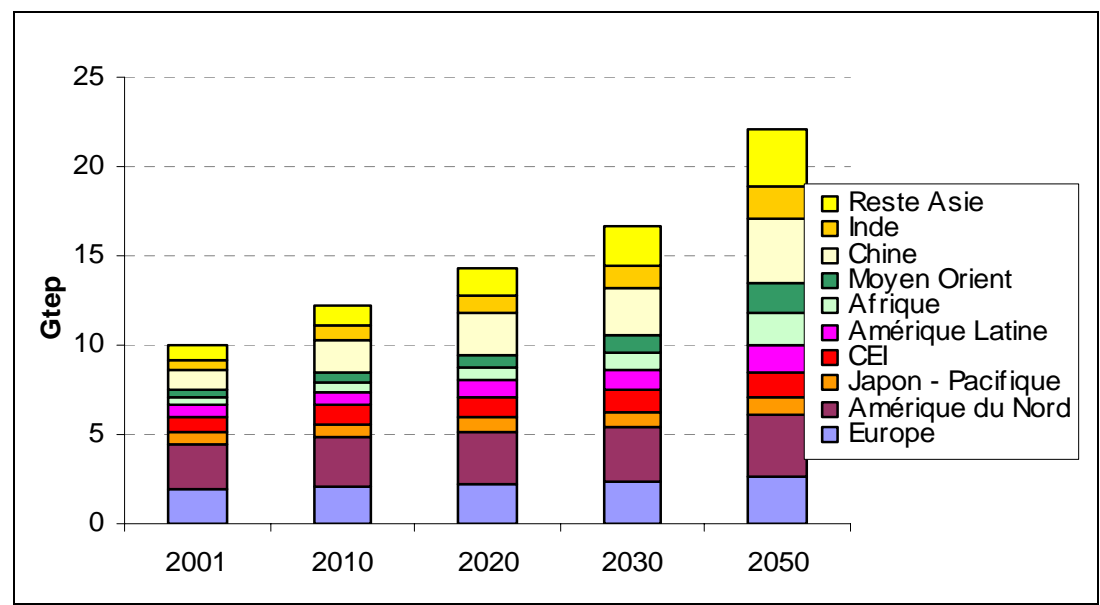

Gtep = milliards de tonnes-équivalent-pétrole

Source : modèle POLES 
Du côté de l'offre, la production de pétrole conventionnel augmente jusqu'en 2030 puis elle se stabilise et commence à décliner vers 2050 (figure 4). Le modèle simule donc un pic pétrolier assez plat, mais à un niveau bas, qui n’est supérieur que de $20 \%$ à la production actuelle. Pour permettre cette stabilisation de la production conventionnelle mondiale, les pays du Golfe arabo-persique doivent plus que doubler leur production. La croissance de la consommation mondiale, ralentie par les prix élevés, est alors essentiellement couverte par la montée des huiles non conventionnelles (pétroles ultra-lourds et sables asphaltiques) qui progressent rapidement à partir de 2030.

Figure 4 : Production de pétrole (2001-2050), scénario tendanciel

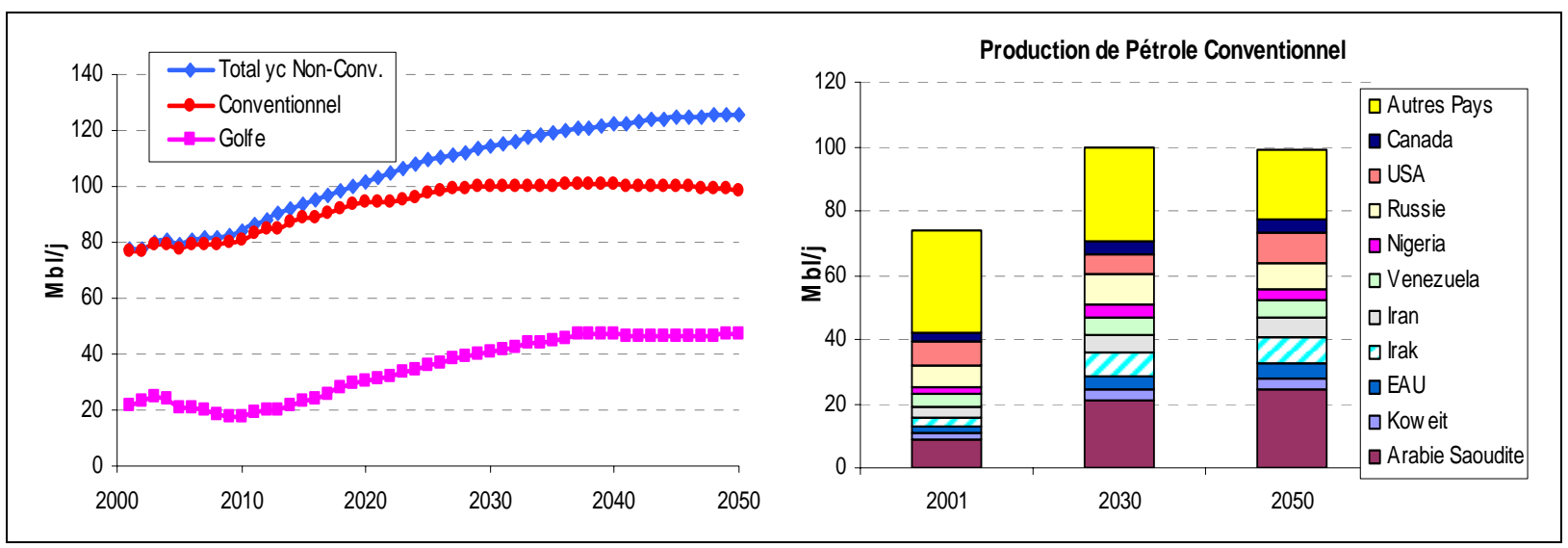

$\mathrm{Mbl} / \mathrm{j}=$ millions de barils par jour

Source : modèle POLES

Conséquence de ces dynamiques de la consommation mondiale et des contraintes de production, le prix du pétrole augmente sur la période pour atteindre en 2050 plus de 100 \$, en prix d'aujourd'hui (figure 5). Le prix du gaz suit une évolution similaire, à un niveau cependant inférieur qui reflète une contrainte de ressource relativement moins forte, ou plutôt décalée dans le temps, et une décote due au coût des infrastructures de transport-distribution.

Figure 5 : prix internationaux du pétrole et du gaz (scénario tendanciel sur 2005-2050)

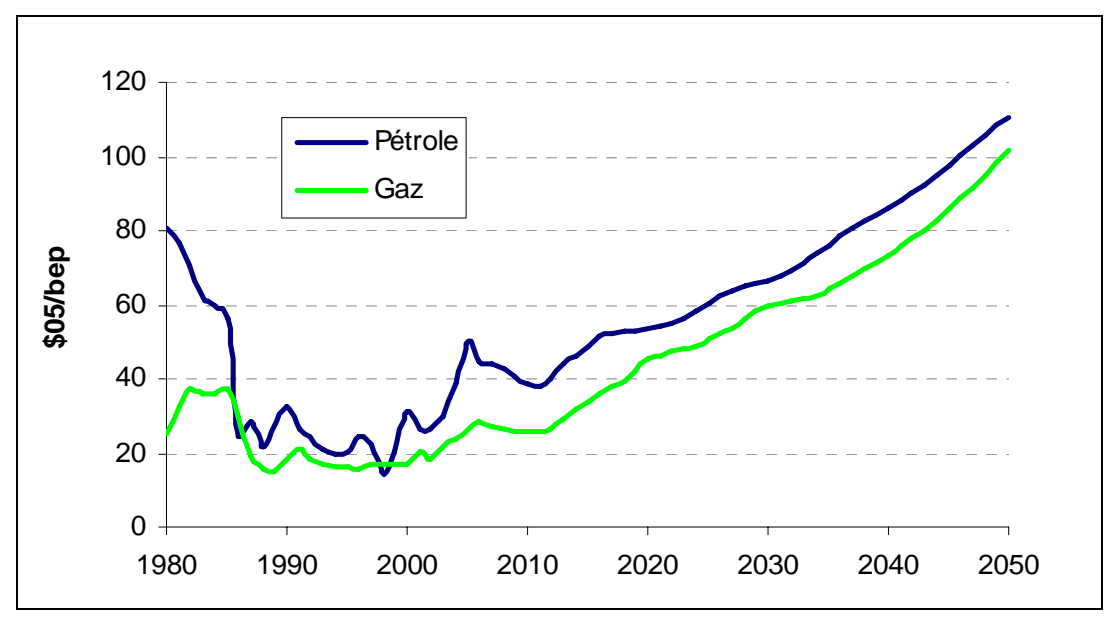

bep = baril-équivalent-pétrole

Source : modèle POLES 
Les émissions de CO2, quant à elles, augmentent de plus de $90 \%$ entre 2000 et 2050, légèrement moins rapidement que la consommation primaire de combustibles fossiles (+95\%), du fait de la montée des renouvelables et du nucléaire ainsi que de la capture et stockage du CO2. Cette option de capture et stockage du CO2 se développe en effet au-delà de 2030, poussée par une valeur-carbone qui la rentabilise dans certaines régions. La répartition géographique des émissions de CO2 s’inverse (figure 6). Les pays industrialisés, sources de plus de la moitié des émissions mondiales de CO2 jusqu'en 2010, voient leur part ramenée à environ 30 \% en 2050. Alors même qu'il s’agit d'un scénario tendanciel, leurs émissions en volume se stabilisent : sous l'effet du déclin démographique, du ralentissement économique et de valeurs-carbone croissantes, ces émissions, après avoir augmenté en valeur absolue jusqu'en 2030, décroissent au-delà de cette date et retrouvent en 2050 leur niveau du début des années 2000.

\section{Figure 6 : répartition géographique des émissions de CO2 (2000-2050), scénario tendanciel}

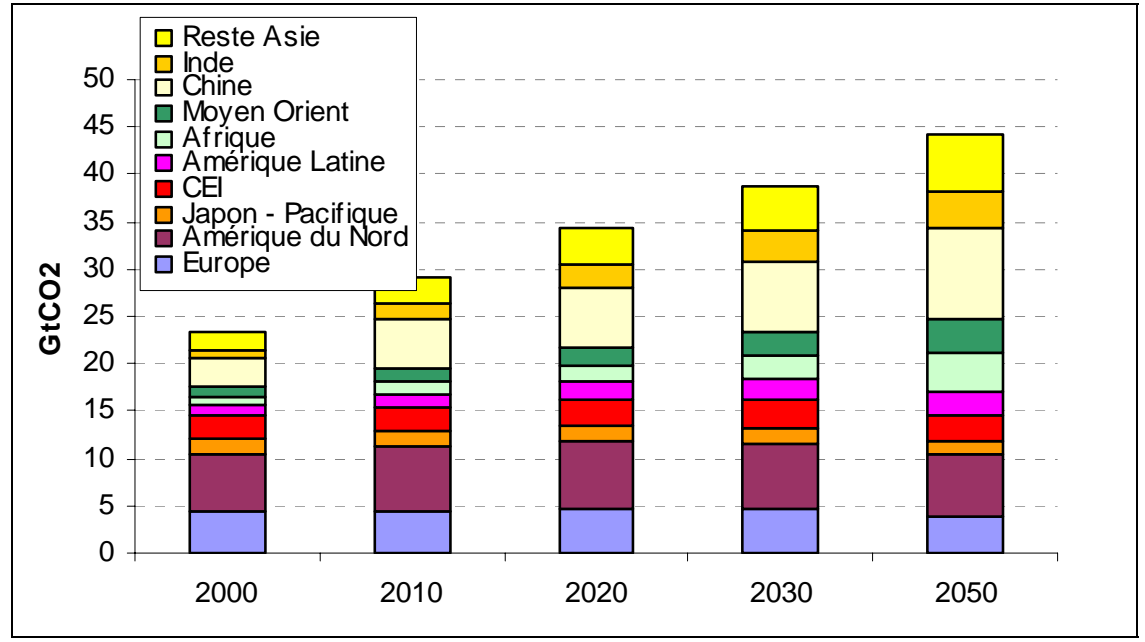

GtCO2 = milliards de tonnes-équivalent-gaz carbonique

Source : modèle POLES

\subsection{Propos d'étape}

Malgré l'introduction de valeurs-carbone dans le modèle, afin de simuler la mise en place d'actions de réduction d'émissions de CO2, le scénario énergétique mondial tendanciel fait clairement apparaître, à l'horizon 2050, une poursuite de la croissance des consommations d'énergie, y compris celles des énergies fossiles. Le résultat est un quasi-doublement des émissions de CO2 en 2050, qui serait clairement incompatible avec des scénarios de maîtrise du changement climatique puisqu'il conduirait à des profils de concentration de l'ordre de 900 à 1000 ppmv.

Le prix du pétrole, quant à lui, s’établit en fin de période autour de 100 \$ en prix constants. Ce niveau est bien plus élevé que le coût de développement des liquides non conventionnels, pétroles techniques, ultra-lourds, sables asphaltiques, schistes bitumineux et même liquides de synthèse à partir du charbon. C'est d'ailleurs pour cette raison que la projection fait apparaître une forte proportion d'huiles non-conventionnelles. Ce niveau de prix est requis non seulement pour stimuler l'offre, mais aussi pour freiner significativement la demande mondiale. Le prix doit alors dépasser le coût de développement des alternatives (comme c’est déjà le cas aujourd’hui). 
Le scénario tendanciel est sans doute plausible compte tenu des dynamiques et contraintes du système. Mais il est également non désirable du point de vue des considérations d'environnement global, puisqu'il conduirait à des niveaux de concentration et donc de changement climatique très importants.

Les scénarios développés pour explorer des futurs alternatifs pour l'énergie et le changement climatique présentent tous des caractéristiques communes : lorsque des objectifs climatiques conformes à l'Article 2 de la Concention Cadre sur les Changements Climatiques sont retenus, il faut diviser les émissions des pays industrialisés par un facteur deux à quatre d'ici 2050 et considérablement limiter la progression des émissions dans les pays en développement ${ }^{5}$. Dans la mesure où ce type de scénarios inspire aujourd'hui les objectifs climatiques à long terme de plusieurs pays européens, il convient d'en explorer les implications majeures pour le système énergétique mondial.

\section{Un scénario de forte contrainte carbone : stabilisation à $450 \mathrm{ppmv}$ et facteur 4 dans les pays industrialisés}

Le scénario tendanciel présenté précédemment apporte une image plausible des évolutions énergétiques et des émissions de CO2 à l'horizon 2050, dans l'hypothèse où les tendances majeures actuelles se poursuivent et où les politiques climatiques resteraient limitées. A l'inverse, dans le scénario alternatif proposé ci-dessous, une contrainte d'émission est déterminée a priori et le modèle identifie la valeur-carbone qui permettrait d'atteindre l’objectif retenu. Il convient ici de souligner que cette valeur-carbone est en quelque sorte une « variable-proxy » qui synthétise l'ensemble des incitations (taxes, quotas d'émission, politiques et mesures) susceptibles d'être combinées pour obtenir les changements désirés, dans une problématique de type « dose-réponse ». Elle traduit donc l'intensité des politiques à mettre en œuvre, mais on ne peut à ce stade en inférer un niveau précis de taxe ou un prix de marché des quotas.

Le scénario proposé explore un cas de contrainte carbone extrêmement forte, mais qui répondrait globalement à l'objectif européen de limiter la hausse des températures moyennes à moins de $+2^{\circ} \mathrm{C}$ par rapport à l'ère pré-industrielle ${ }^{6}$. Il s'agit alors de s'inscrire au plan mondial sur une trajectoire de stabilisation à $450 \mathrm{ppmv}$, ce qui implique : i. un plafonnement des émissions mondiales avant 2020, ii. un retour au niveau actuel d'émission avant 2030 et enfin iii. des émissions en 2050 ramenées à la moitié de ce niveau actuel (figure 7, Courbe WRE 450). Pour atteindre ce profil, lorsque le même signal en terme de valeur-carbone est appliqué à toutes les régions du monde, alors les réductions requises dans les pays industrialisés sont bien une division par un facteur quatre des émissions en 2050, par rapport à 1990. Ceci confirme la grande cohérence, au moins au niveau des objectifs, des politiques explorées aujourd'hui en Europe autour du triptyque : moins de $2^{\circ} \mathrm{C}$ d'augmentation des températures, stabilisation des concentrations de CO2 à 450 ppmv, réductions facteur 4 dans les pays industrialisés. Dans les pays en développement les réductions sont moins importantes, elles sont tout de même notables, de l’ordre de 10 \% en moyenne, malgré le doublement de leur consommation d'énergie entre 2000 et 2050 (voir infra).

\footnotetext{
${ }^{5}$ Parmi les études prospectives qui explorent de tels scénarios, on peut citer (Enerdata-LEPII-EPE, 2005 ; Radanne, 2004 ; Prévot, 2004) pour la France

${ }^{6}$ Objectif énoncé en 1996 au $1939{ }^{\text {ème }}$ Conseil de l'Environnement de l'Union européenne, et confirmé par le Conseil Européen des 22-23 mars 2005, sur la base de (Commission des Communautés Européennes, 2005).
} 
Figure 7 : Profils de stabilisation des émissions d'après le GIEC ${ }^{7}$

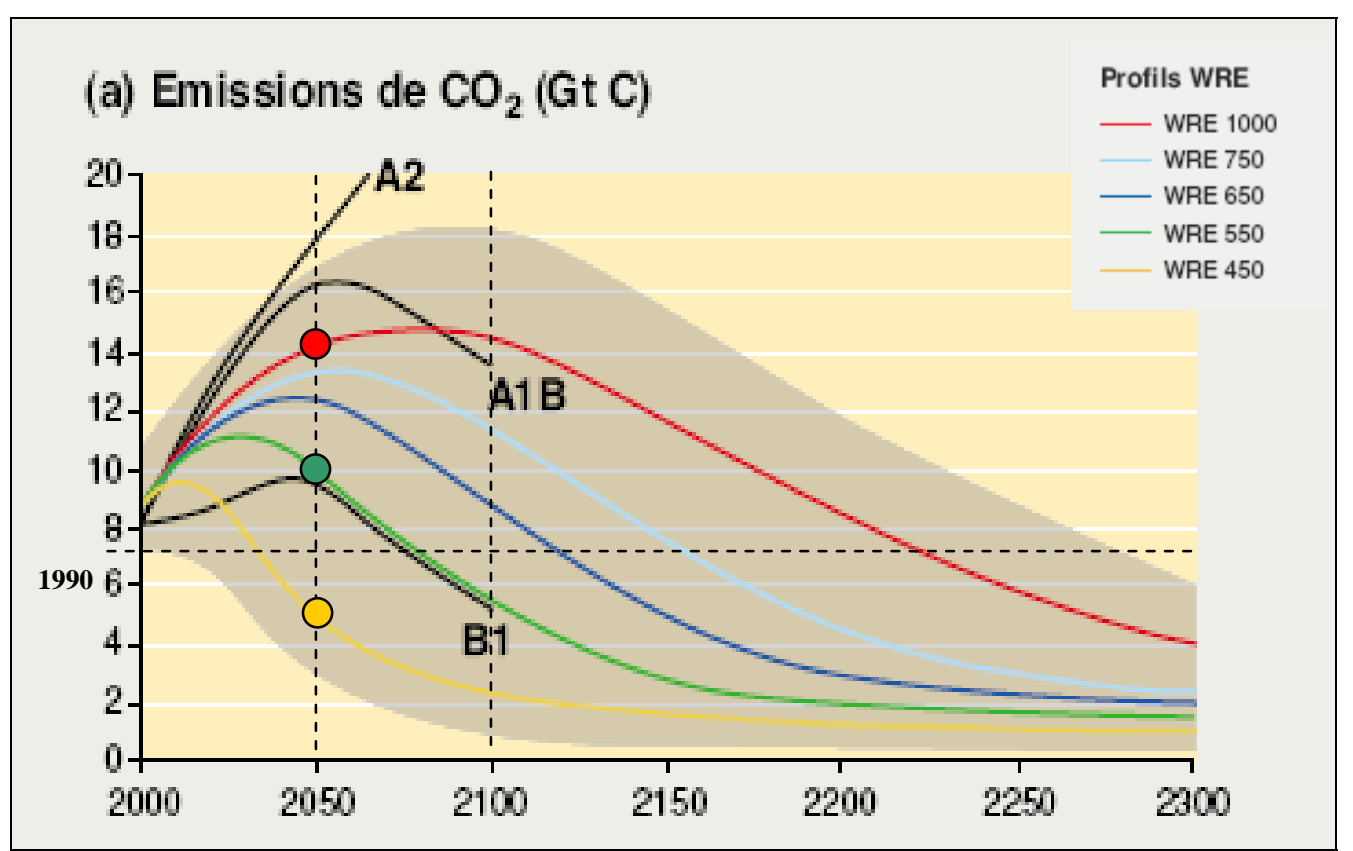

Source: Figure 6-a du Résumé du Troisième Rapport d'Evaluation du GIEC (IPCC, 2001), complétée par les auteurs $^{89}$

La figure 8 montre le niveau de la taxe-carbone nécessaire pour atteindre les objectifs de réductions d'émissions. Les valeurs obtenues, de plusieurs centaines d'Euro par tonne de CO2 apparaissent extrêmement élevées. Cependant, on l’a noté plus haut, elles ne font que témoigner de l'intensité des politiques à mettre en œuvre. Les résultats obtenus pour la valeurcarbone fournissent deux types d'indications, qui seront confirmées plus loin par l'analyse des résultats détaillés. Premièrement, il apparaît que pour respecter le profil de forte décroissance des émissions après le plafond, les fonctions de réaction du modèle imposent une croissance rapide de la valeur du carbone, alors que les simulations avec une croissance linéaire de cette valeur ne permettent qu'un ralentissement de l'augmentation des émissions. Deuxièmement, cette forte croissance montre qu'il n'y a pas, dans la description du système énergétique offerte par le modèle POLES, de « technologie-limite » (backstop technology) qui permettrait, à partir d'une certain seuil de valeur-carbone, des réductions massives ; aux niveaux de valeurs du carbone considérées, les solutions technologiques d'offre à bas contenu carbone sont toutes rentables, mais leurs potentiels de développement sont néanmoins contraints ; il faut alors poursuivre la hausse des valeurs du carbone élevées pour peser sur la consommation d’énergie et favoriser les technologies d’usage Très Basses Emissions.

\footnotetext{
${ }^{7}$ Sur cette figure, les émissions sont exprimées en GtC (milliards de tonnes-équivalent-carbone), alors qu’elles sont en GtCO2 (milliards de tonnes-équivalent-gaz carbonique) sur les autres figures. $1 \mathrm{GtCO}=44 / 12 \mathrm{GtC}$ ${ }^{8}$ Le profil d'émissions décrit prend aussi en compte le CO2 de la déforestation, pour environ 1 GtC.
} 
Figure 8 : Taxe-carbone nécessaire à la réalisation des objectifs (2000-2050)

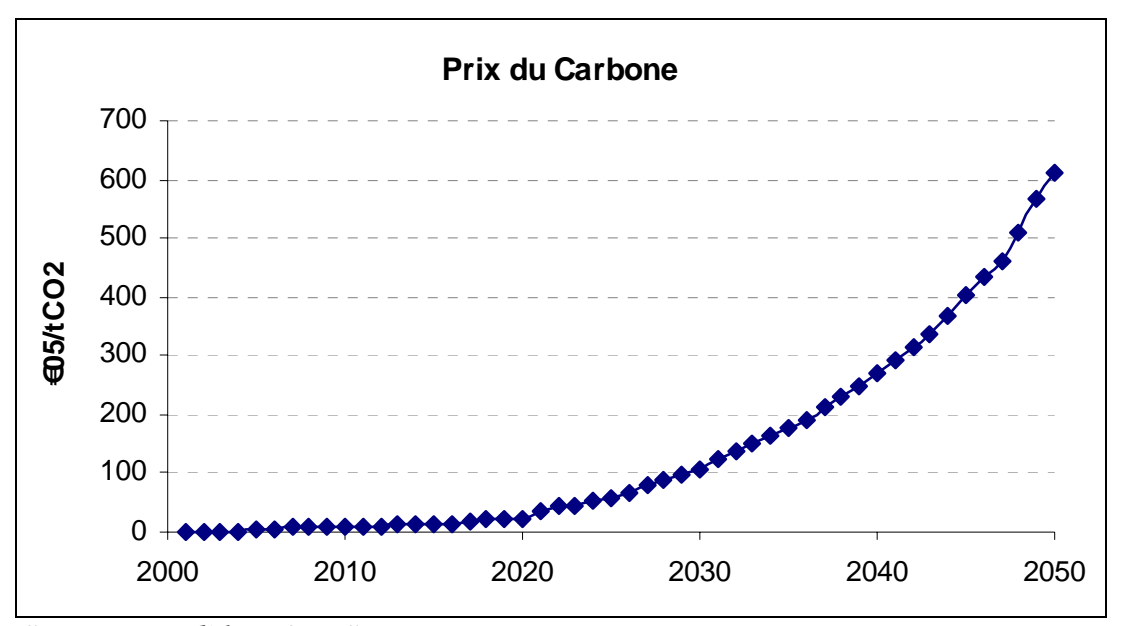

Source : modèle POLES

La taxe-carbone élevée produit l'effet attendu sur les émissions de CO2 (figure 9). Celles-ci sont globalement divisées par 2 entre 2000 et 2050, avec une division par 4 dans les pays industrialisés et une diminution d'environ 10 \% dans les pays en développement.

Figure 9 : émissions de CO2 (2000-2050), scénario « Stabilisation 450 - Facteur 4 »

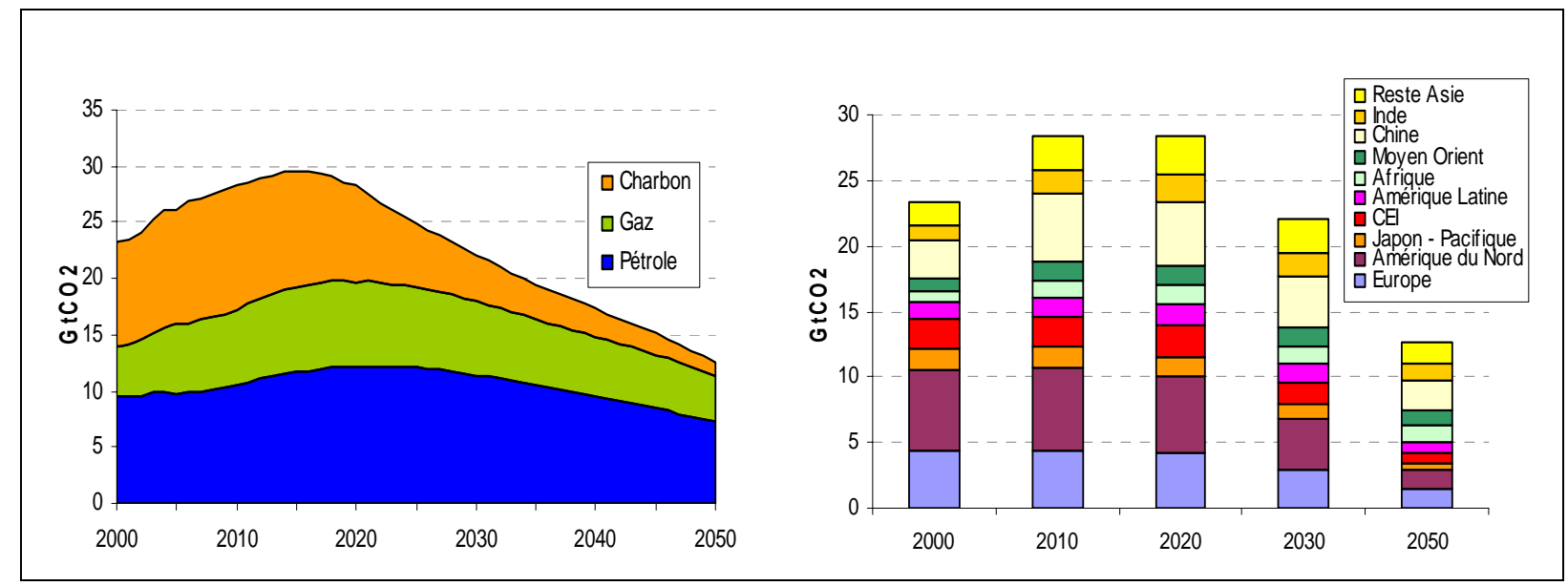

GtCO2 = milliards de tonnes-équivalent-gaz carbonique

Source : modèle POLES

Quelles sont les conséquences de ce scénario sur le système énergétique mondial ? La section 3.1 expose les incidences sur les grands équilibres énergétiques (demande-offre). La section 3.2 met en lumière les changements technologiques induits, en particulier au niveau de la production d'électricité.

\subsection{Conséquences sur les grands équilibres énergétiques}

Comparativement au scénario tendanciel où les consommations d'énergie sont multipliées par deux, dans le scénario « Stabilisation 450 - Facteur 4 » les consommations mondiales d'énergie cessent de croître à partir de 2020 puis se stabilisent globalement à un niveau environ $30 \%$ supérieur à celui des années 2000. L'introduction d'une taxe-carbone élevée 
agit en particulier sur les consommations d'énergies fossiles : ces dernières représentent moins de la moitié du bilan en 2050 suite à des substitutions énergétiques en faveur des énergies non émettrices de CO2 (renouvelables, nucléaire, figure 10).

Figure 10 : Consommation totale mondiale et répartition par sources (2001-2050), scénario « Stabilisation 450 - Facteur 4 »

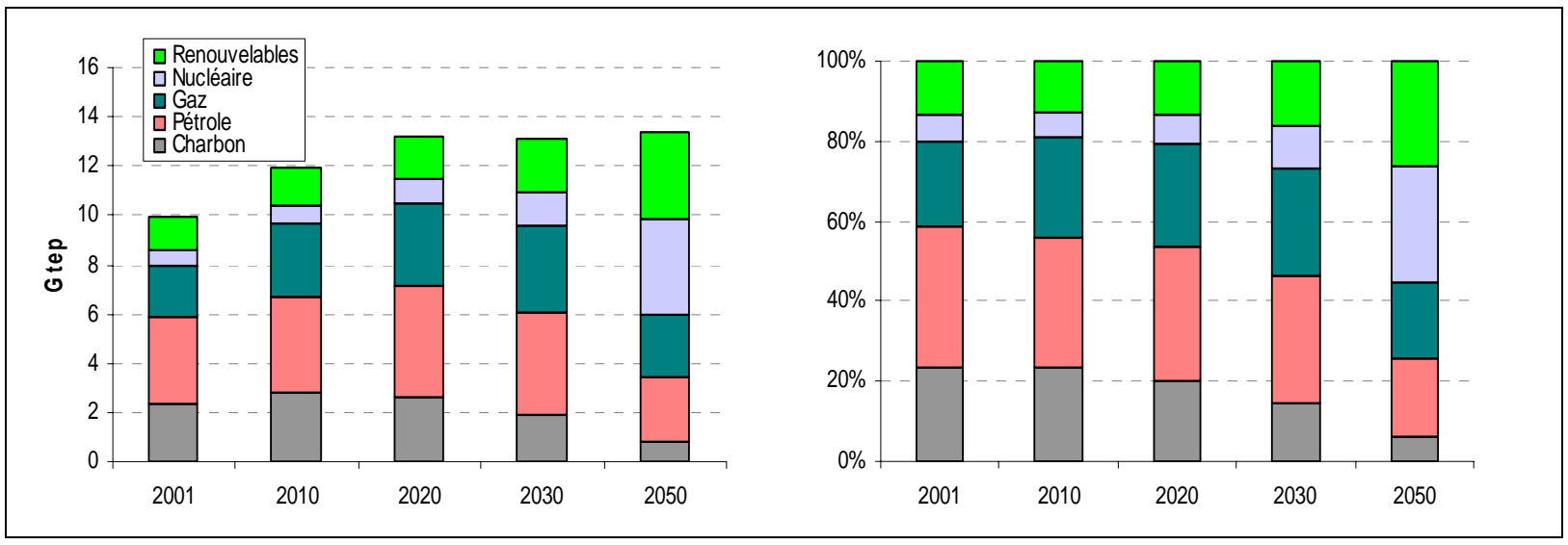

Gtep = milliards de tonnes-équivalent-pétrole

Source : modèle POLES

En termes de répartition géographique (figure 11), les consommations des pays industrialisés croissent légèrement jusqu'en 2020 puis régressent pour atteindre un niveau inférieur à celui de 2001. A l'inverse, dans les pays en développement, les consommations augmentent sur toute la période, mais de façon beaucoup plus lente que dans le scénario tendanciel. Au total la consommation des pays en développement est multipliée par deux, de 4 à 8 Gtep en 2050 . Cette augmentation traduit le fait que l'accès à des sources d'énergie modernes (par opposition aux sources traditionnelles que sont le bois de feu, les déchets végétaux et animaux) demeure un élément-clé de la réduction de la pauvreté et du développement humain, même dans le cas extrême étudié ici (DFID, 2002 ; UN, 2005).

Figure 11 : répartition géographique de la consommation mondiale (2001-2050), scénario « Stabilisation 450 - Facteur 4 »

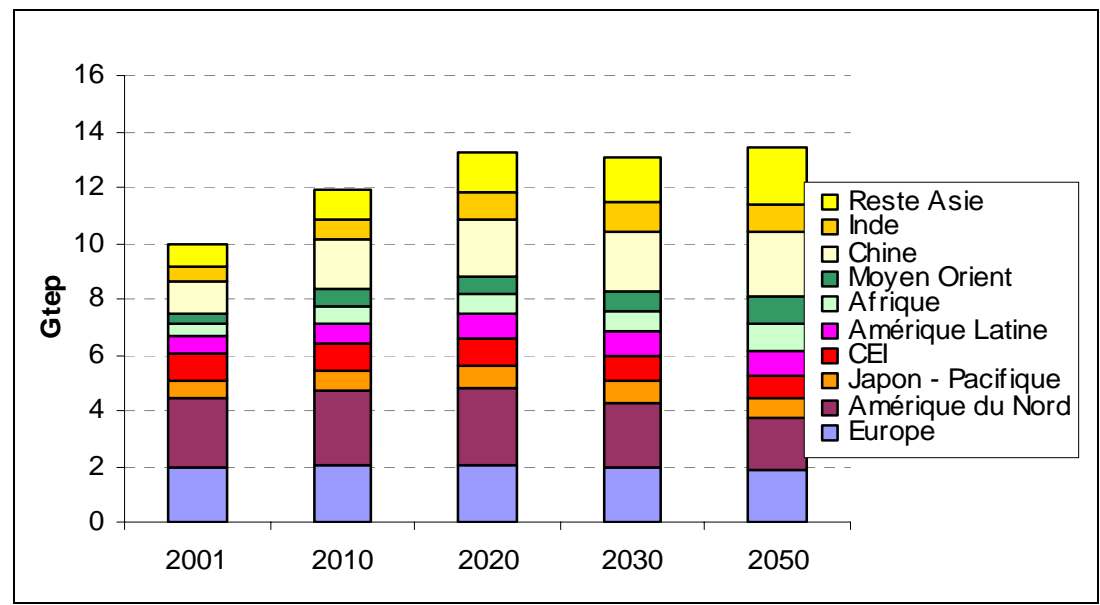

Gtep = milliards de tonnes-équivalent-pétrole

Source : modèle POLES 
Du côté de l'offre, la production de pétrole est sensiblement équivalente à celle du scénario tendanciel jusqu'en 2020, du fait de taxes-carbone d'un ordre de grandeur comparable sur cette première période (figure 12). En revanche, la production diminue au-delà de 2020 dans le scénario « Stabilisation 450 - Facteur 4 », sous l'influence de la taxe-carbone élevée et toujours croissante qui pèse sur les consommations.

Figure 12 : production de pétrole (2001-2050), scénario « Stabilisation 450 - Facteur 4 »

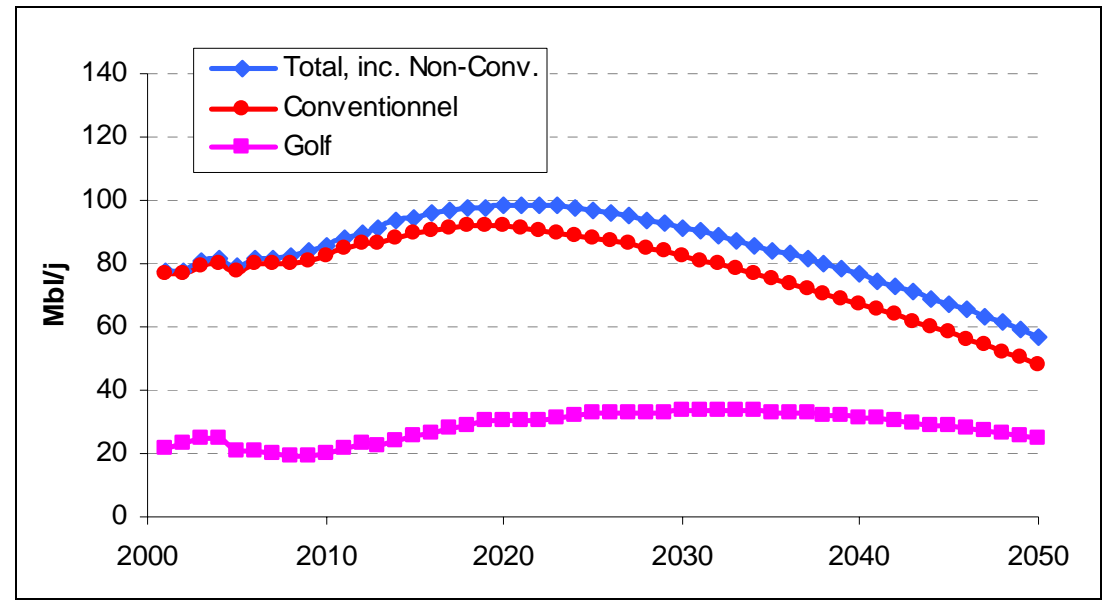

$\mathrm{Mbl} / \mathrm{j}=$ millions de barils par jour

Source : modèle POLES

Le prix du pétrole et celui du gaz sur les marchés internationaux sont jusqu'à deux fois inférieurs aux niveaux qu'ils atteignent dans le scénario tendanciel au-delà de 2020 (figure 13). Cependant il convient de noter que ces infléchissements de prix concernent les producteurs de pétrole et de gaz, mais ne sont pas répercutés dans les prix aux consommateurs finaux, dans la mesure où ces derniers sont soumis à la taxe-carbone. C'est d'ailleurs le niveau très élevé de la taxe-carbone qui entraîne une diminution considérable de la demande en énergies fossiles, réduit les tensions sur les ressources pétrolières et gazières et rend cellesci moins coûteuses à exploiter.

Figure 13 : prix du pétrole (2001-2050), scénario « Stabilisation 450 - Facteur 4 »

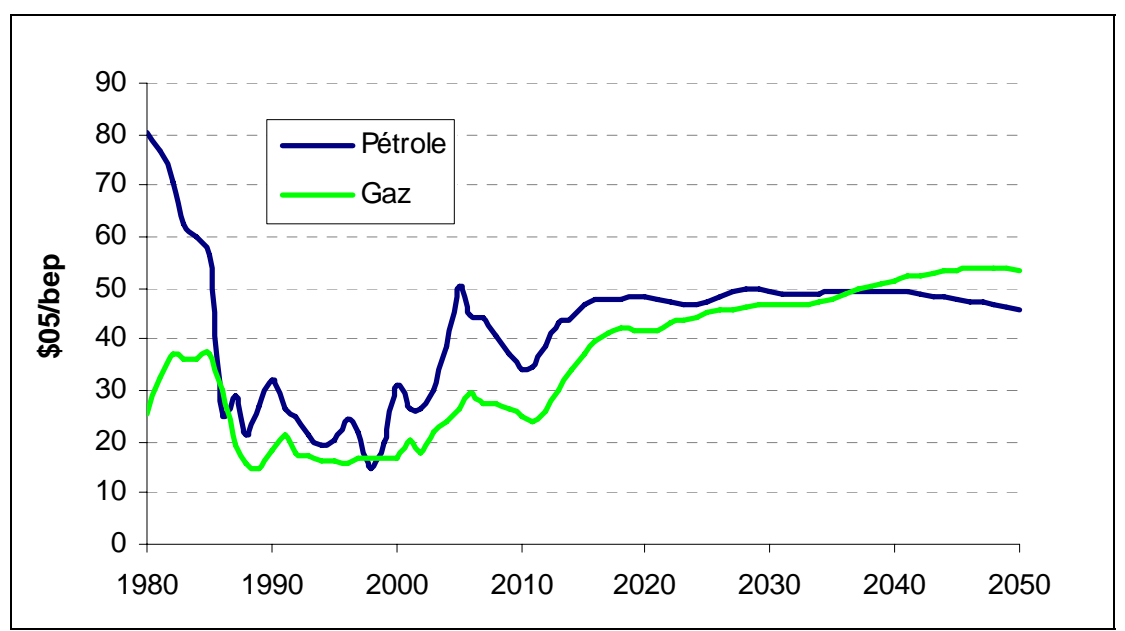

bep=baril-équivalent-pétrole

Source : modèle POLES 
L’une des principales conclusions à tirer de la comparaison du scénario tendanciel et du scénario exploratoire « Stabilisation 450 - Facteur 4 » est la suivante : dans le scénario tendanciel, la raréfaction croissante du pétrole ne limite que faiblement la croissance des consommations d'énergie, elle entraîne en revanche un retour marqué du charbon, probablement catastrophique pour le climat global ; inversement dans les scénarios de forte contrainte carbone et du fait de l'auto-limitation des consommations, le pétrole redevient relativement plus abondant et meilleur marché. Il y a là manifestement un effet « gagnantgagnant » des politiques climatiques, peut-être plus important que tous ceux identifiés jusqu'à aujourd'hui (alors même que le scénario tendanciel est de ce point de vue « perdantperdant »).

\subsection{Changements technologiques induits par le scénario}

Les réductions d'émissions peuvent provenir de deux grands volets d'actions dans le système énergétique, un volet portant sur la demande d'énergie et un autre sur l'offre, en particulier dans le système électrique. Les actions sur la demande d'énergie comprennent des substitutions entre énergies favorisant les moins émettrices ${ }^{10}$ ainsi que, à plus long terme, une maîtrise accrue de la consommation via le développement et la diffusion d'équipements Très Basses Emissions («TBE ») dans le bâtiment, les transports et l’industrie.

Le deuxième type d'actions porte sur quatre options différentes de « décarbonisation » de la production d'électricité. Cette décarbonisation est rendue possible par les substitutions entre énergies fossiles (abandon du charbon ou du pétrole au profit du gaz, sans technologie de séquestration) et par trois options technologiques distinctes : le recours accru aux énergies renouvelables (avec les énergies-flux, éolienne, solaire et hydraulique ou la biomasse) ; le développement de l'énergie nucléaire (avec les réacteurs de technologie actuelle et l'arrivée de réacteurs dits de quatrième génération); et enfin la capture et stockage du $\mathrm{CO} 2$, pour les installations de grande puissance dans la production thermique d’électricité ${ }^{11}$.

Dans le scénario « Stabilisation 450 - Facteur 4 », ces divers types d'action sont tous mobilisés pour atteindre l’objectif de réductions des émissions de CO2 (figure 14). Mais ils contribuent aux réductions à des degrés différents selon les périodes. Dans un premier temps, les réductions proviennent essentiellement des substitutions entre combustibles fossiles, à la fois du côté demande (dans les usages où les combustibles fossiles sont substituables facilement), et du côté de la production d'électricité. Puis les technologies de production d'électricité moins émettrices (capture et stockage, renouvelables, nucléaire) se développent suffisamment pour assurer une grande part des réductions jusqu’en 2035. En fin de période, la pénétration des technologies de demande « Très Basses Emissions » (dont le temps de déploiement est lié en particulier à la longue durée de vie des bâtiments) redevient le principal contributeur aux réductions.

Il faut noter que les énergies renouvelables et le nucléaire contribuent de façon croissante à l'effort de réduction alors que l'impact des technologies de capture et séquestration diminue en fin de période du fait d'un coût croissant d'accès aux sites de stockage et des pertes de $\mathrm{CO} 2$ au moment de la capture qui finissent par rendre ces options technologiques sensibles à la taxe-carbone lorsqu'elle atteint des niveaux importants.

\footnotetext{
101 tep de charbon entraîne lors de sa combustion en moyenne l'émission d'une tonne de carbone (tC), 1tep de pétrole environ 3/4 tC, 1 tep de gaz naturel 2/3 tC

${ }^{11}$ La capture et le stockage du CO2 peuvent aussi être mobilisés dans l’industrie.
} 
Figure 14 : contribution annuelle des divers types d'action de réduction des émissions de CO2 (2010-2050), scénario « Stabilisation 450 - Facteur 4 »
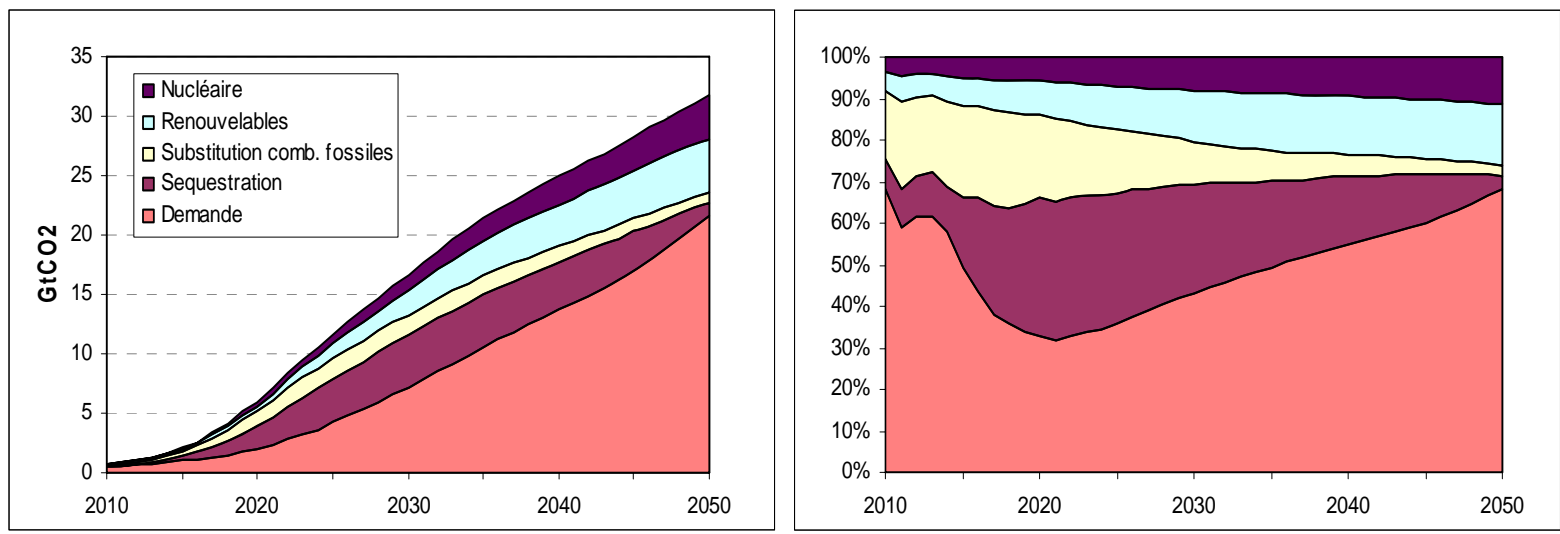

GtCO2 = Milliards de tonnes-équivalent-gaz carbonique

Source : modèle POLES

L'analyse des contributions cumulées des diverses actions sur 2010-2050 montre la contribution prépondérante des actions sur la demande, suivie de la capture et stockage du carbone, du développement des énergies renouvelables, et à parts égales, de l'augmentation de la production d'électricité nucléaire et de la substitution entre combustibles fossiles (figure 15). Il faut ici noter que ces contributions sont mesurées par rapport à la projection tendancielle, ce qui explique pourquoi la contribution incrémentale de la capture et stockage est importante (elle est très faible dans la projection tendancielle) ou pourquoi celle des renouvelables est supérieure à celle de l'énergie nucléaire, alors qu'en valeur absolue la contribution de l'énergie nucléaire au bilan énergétique mondial est dans les deux cas légèrement supérieure à celle des renouvelables.

Figure 15 : contributions cumulées aux réductions d'émissions de CO2 (2010-2050, 180 GtC), « Stabilisation 450 - Facteur 4 »

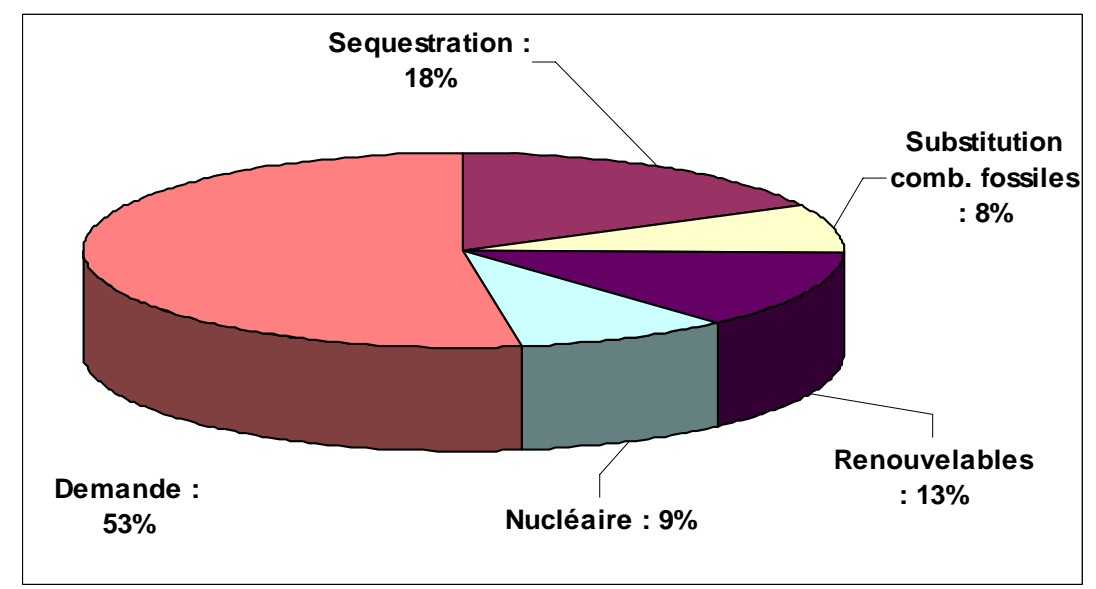

GtCO2 = Milliards de tonnes-équivalent-gaz carbonique

Source : modèle POLES 


\section{Conclusion}

En conclusion, la comparaison du scénario tendanciel et du scénario « Stabilisation 450 Facteur 4 », montre que la réduction drastique des émissions de GES à l'horizon 2050 par rapport à la progression tendancielle n'est réalisable qu'au prix de politiques très actives, impliquant une valeur carbone élevée et fortement croissante sur la période, permettant le développement massif des technologies Très Basses Emissions. Ces politiques ne seront probablement acceptables que si par ailleurs est mis en œuvre un ensemble de politiques et mesures qui permettront, d'une part de réduire le signal-prix nécessaire, et d'autre part de le rendre supportable aux citoyens/consommateurs et aux entreprises.

Ce scénario « Stabilisation 450 - Facteur 4 », même s’il est avant tout un scénario exploratoire, fait apparaître qu'aucune grande option technologique ne peut être négligée, si l'on souhaite atteindre un niveau d'émissions global compatible avec la stabilisation de la concentration des GES à un niveau compatible avec l'objectif climatique européen. Ces grandes options sont bien : les technologies de consommation «Très Basses Emissions » dans le bâtiment, les transports et l’industrie, les énergies renouvelables en déploiement massif , mais aussi l'énergie nucléaire de troisième et quatrième générations et la capture et stockage du carbone.

Le prix du carbone influe directement sur les émissions de GES mais aussi sur les prix des énergies, et en particulier le prix du pétrole : entre le scénario tendanciel et le scénario «Stabilisation 450 - Facteur 4 », le prix du pétrole est divisé par deux et il se stabilise aux niveaux d'aujourd'hui. De fait, une taxe-carbone très élevée entraîne une diminution de la demande pour tous les combustibles fossiles, en particulier pour le charbon, mais aussi pour le pétrole. La tension sur la ressource en pétrole conventionnel se relâche par rapport au scénario tendanciel et entraîne une moindre dépendance énergétique mondiale par rapport à la région du Golfe.

Il apparaît ainsi que des scénarios de politiques climatiques ambitieuses visant à limiter le changement climatique permettraient aussi de gérer la question de l'épuisement des ressources mondiales d'hydrocarbures. Notre exercice a donc permis de montrer que la recherche d'un développement énergétique durable repose largement sur l'ambition et l'effectivité de ces politiques. Il comporte évidemment des limites. La première d'entre elles a trait à l'absence de rétro-action de la contrainte carbone sur l'économie : le modèle POLES est en effet un modèle d'équilibre partiel qui ne permet pas de représenter les impacts macroéconomiques de politiques climatiques ambitieuses. La seconde résulte d'une représentation encore incomplète des changements structurels que l'adoption d'une trajectoire à bas profil d'émission déclencherait dans l'économie, les technologies et les comportements. Sur ces deux plans, les recherches sont en cours : par dialogue du modèle sectoriel avec des modèles économiques et par un effort de représentation plus détaillée des caractéristiques possibles d'une « société Facteur 4 ». 


\section{Références bibliographiques}

Commission des Communautés Européennes, 2005. Vaincre le changement climatique planétaire. Communication de la Commission au Conseil, au Parlement Européen, au Comité Economique et Social Européen et au Comité des Régions, SEC(2005) 180.

Criqui, P., Kitous, A., Berk, M., et al., 2003. Greenhouse gas reduction pathways in the UNFCCC process up to 2025, LEPII-EPE, RIVM, ICCS-NTUA, CES-KUL, Union européenne. DG Environnement.

http://europa.eu.int/comm/environment/climat/pdf/pm_techreport2025.pdf

Criqui, P., Kouvaritakis, N., 2000. “World energy projections to 2030”, International journal of global energy issues, Vol. 14, n.1/2/3/4, p. 116-136.

Criqui,P., Viguier, L., 2000. "Kyoto and technology at world level : costs of CO2 reduction under flexibility mechanisms and technical progress", International journal of global energy issues, Vol. 14, n.1/2/3/4, p. 155-168.

Department of Trade and Industry of the United Kingdom (DTI), 2003. Energy White Paper : our energy future-creating a low carbon economy. février.

Enerdata, LEPII-EPE, 2005. Etude pour une prospective énergétique concernant la France, février.

http://www.industrie.gouv.fr/energie/prospect/textes/prosp-jr-2030-2050.htm

Department for International Development (DFID), 2002. Energy for the Poor. Underpinning the Millennium Development Goals.

http://www.dfid.gov.uk/pubs/files/energyforthepoor.pdf

IPCC (Intergovernmental Panel on Climate Change), 2001a. Summary for Policymakers to Climate Change 2001: Synthesis Report of the IPCC Third Assessment Report.

http://www.ipcc.ch/pub/un/syreng/spm.pdf

Porter, E., 1995. Are we running out of oil ?, American Petroleum Institute, Washington, December, Discussion paper ; 81.

Prévot, H., 2004., Diviser par trois en trente ou quarante ans nos émissions de CO2 http://www.2100.org/PrevotEnergie/

Radanne, P., 2004. « La division par quatre des émissions de CO2 en France d’ici 2050 », Reponsabilité et environnement, n. 33, janvier, p. 13-29.

http://www.effet-de-serre.gouv.fr/fr/etudes/Facteur4-franc\%20BAT.pdf

United Nations, 2005. The Energy Challenge for Achieving the Millenium Development Goals, UN-Energy.

United Nations Framework Convention on Climate Change (UNFCCC),1992. New York, May 9. 
United Nations Framework Convention on Climate Change (UNFCCC), 1997. Kyoto Protocol to the United Nations Framework Convention on Climate Change.

Wells, P.R.A., 2005. “Oil supply challenges”, Oil and gas journal, vol. 103, n. 7, 21 février, p. 20-28. 